\title{
Opiniones hacia las prácticas anticonceptivas en estudiantes de la universidad del Magdalena, Santa Marta (Colombia)
}

\section{Reviews to contraceptive practices among students at the university of magdalena, Santa Marta (Colombia)}

\author{
Lilibeth Pedraza, ${ }^{1}$ Ximena Socarrás, ${ }^{2}$ Rafael Sevilla, ${ }^{3}$ José López, ${ }^{4}$ Jennifer Jiménez ${ }^{5}$
}

\begin{abstract}
RESUMEN
Introducción: Con los años los problemas de salud pública y social han aumentado de manera alarmante, dentro de los que se destacan el alce de embarazos no deseados, enfermedades de trasmisión sexual, entre otras, así como el poco o inadecuado uso de métodos de planificación. Objetivo: Determinar la opinión de los estudiantes del programa de Medicina de la Universidad del Magdalena, sobre los métodos anticonceptivos. Métodos: El tipo de investigación fue descriptivo de cohorte trasversal, se usó el tipo de muestreo intencional, con una participación de 254 estudiantes. Resultados: El método anticonceptivo de mayor demanda es la vasectomía, seguido por su contra parte femenina, la ligadura de trompas, al mismo tiempo, se observa que el método de la retirada o interrupción es el menos recomendable para poner en práctica. Conclusiones: El preservativo y las pastillas siguen siendo los favoritos al momento de escoger algún método eficaz, mientras que no ven como opción viable el método del ritmo, el calendario o la abstinencia.
\end{abstract}

Palabras Claves: Métodos anticonceptivos, opiniones, ETS, embarazos no deseados.

\begin{abstract}
Introduction: Over the years the problems of social and public health have increased alarmingly, among which stand out the moose unwanted pregnancies, sexually transmitted diseases, among others, as well as little or inappropriate use of planning methods. Objectives: To determine the opinion of students of Medicine program at the University of Magdalena, about contraception. Methods: The research was descriptive cross-cohort, type of purposive sampling was used, with a participation of 254 students. Results: Showed that the increased demand for contraception is vasectomy, followed by his female counterpart, tubal ligation at the same time, it appears that the method of withdrawal or discontinuation is recommended to put less practice. Conclusions:

\footnotetext{
${ }^{1}$ Psicóloga, Mg en Psicología Organizacional y del trabajo. Universidad del Magdalena. Santa Marta. Colombia.

2 Practicante de Psicología. Universidad del Magdalena. Santa Marta. Colombia.

${ }^{3}$ Estudiante Octavo semestre de Medicina. Universidad del Magdalena. Santa Marta. Colombia.

${ }^{4}$ Estudiante noveno semestre de Medicina. Universidad del Magdalena. Santa Marta. Colombia.

${ }^{5}$ Estudiante Noveno semestre de Medicina. Universidad del Magdalena. Santa Marta. Colombia.

Correspondencia: lilibethpatricia@gmail.com
} 
Condoms and pills are still the favorites when choosing an effective method, while not seen as a viable option the rhythm method, calendar or abstinence.

Keywords: Contraception, reviews, STDs, unwanted pregnancies.

\section{INTRODUCCIÓN}

En la actualidad se viven una serie de problemáticas sociales las cuales, según la UNESCO ha aumentado alarmantemente con los años. Dentro de dichas problemáticas se destaca de manera particular los embarazos no deseados, las enfermedades de trasmisión sexual, que según diferentes autores están dadas por la desinformación o las malas prácticas anticonceptivas (1).

En Colombia, la encuesta nacional de Profamilia (2) reveló que del $48 \%$ de los embarazos ocurridos en el lapso de los últimos cinco años han sido esperados pero un $23 \%$ no lo deseaba, pero solo el $7 \%$ de la población usa métodos de barrera, lo que lleva a inferir que el $93 \%$ restante no utiliza ningún método de prevención a Enfermedades de Transmisión Sexual (ETS).

Profamilia (3) publicó datos alarmantes donde el $19 \%$ de las jóvenes con edades entre 15 y 19 años ya son madres o están en embarazo, otro dato es que solo el $7 \%$ de las mujeres que viven sexualmente activas usa condón, lo que indica que el $93 \%$ restante no está usando ningún método de protección, lo que los deja totalmente expuesto a una enfermedad de transmisión sexual.

Resultados de diferentes investigaciones muestran que el $39 \%$ de la población encuestada utiliza métodos anticonceptivos orales, $21 \%$ operación de la mujer, 20,5\% implantes o dispositivos intrauterinos, solo el $1 \%$ de la población encuestada usa métodos de barrera (4).

Estudios realizados (5), encontraron que de la muestra utilizada el $95 \%$ de la población refirió haber escuchado sobre la anticoncepción de emergencia y el 80 \% la consideró un método útil de planificación; los hombres mostraron más conocimiento que las mujeres al respecto. Por su lado Ospina y Manrique muestran que del $100 \%$ de la población solo el 36,2\% tiene la certeza de evitar un embarazo no deseado, donde los participantes manifestaron falta de conocimiento sobre prácticas y métodos anticonceptivos (6).

Gómez dice que dos de cada diez mujeres reveló no estar de acuerdo con el uso de los anticonceptivos hormonales de emergencia, así como también una gran brecha entre conocimientos, actitudes y prácticas sobre los métodos anticonceptivos de emergencia (7). Mientras que Cáceres, utilizando una muestra de 77 estudiantes, expresa que el 92 $\%$ conoce todos los métodos anticonceptivos, pero de este porcentaje sólo el $55 \%$ hace uso adecuado de los mismos, presentando la misma problemática anterior donde los hombres muestran mayor conocimiento que las mujeres, así mismo el 70 \% manifestó necesitar mayor información sobre el tema (8). 
En una investigación donde se buscó determinar la percepción de los participantes sobre las prácticas sexuales y anticonceptivas se determinó que esta fue positiva y practicaban la misma en responsabilidad como pareja, lo cual es vital para tener una práctica sexual y reproductiva saludable $(9)$.

Resultados obtenidos en diferentes investigaciones donde se encontró que a pesar de que los jóvenes conocen los métodos anticonceptivos sólo un pequeño grupo los utiliza y perciben el embarazo como un aspecto biológico, el embarazo pasa a ser parte de una responsabilidad, más que una consecuencia por una práctica sexual. No obstante, en un estudio realizado en el año 2010 se encontró que las mujeres que participaron poseen una actitud favorable y una opinión neutra respecto a las prácticas anticonceptivas $(9$, 10).

Por lo expuesto anteriormente, se busca en este estudio identificar las opiniones de los estudiantes de Medicina sobre las prácticas anticonceptivas.

\section{MÉTODOS}

El tipo de investigación que se usó fue de tipo cuantitativo descriptivo, con un diseño trasversal. El tipo de muestreo fue intencional. La población estuvo comprendida por 436 estudiantes y la muestra utilizada fue de 254 personas.

Para la recolección de información se utilizó una escala autoaplicada de evaluación de actitudes y opiniones hacia las prácticas anticonceptivas (11), donde solo se tuvieron en cuenta los resultados obtenidos en OPINIONES con un alfa de cronbach:067.

La presente investigación tuvo en cuenta los requisitos exigidos en la Resolución 8430 de 1993 del Ministerio de Salud de Colombia, la cual establece normas científicas, administrativas y técnicas en investigaciones en salud; así como el aspecto bioético contemplado en la Ley 1090 de 2006 para la investigación en psicología $(12,13)$.

\section{RESULTADOS}

En cuanto al género se encontró una media de 1,2 al igual que la moda, lo que indica que el género femenino es el que más se repite. Con respecto a la edad encontramos que hay una media de 20.52 y una moda de 20 lo que indica que la edad que más se repite es 20 años.

TABLA 1: Género

\begin{tabular}{|lc|c|c|c|c|}
\hline & Frecuencia & Porcentaje & Porcentaje válido & $\begin{array}{c}\text { Porcentaje } \\
\text { acumulado }\end{array}$ \\
\hline Válidos & femenino & 192 & 75.6 & 75.6 & 75.6 \\
& masculino & 62 & 24.4 & 24.4 & 100.0
\end{tabular}




\begin{tabular}{|l|l|l|l|l|} 
Total & 254 & 100.0 & 100.0 & \\
\hline
\end{tabular}

En la tabla anterior se pude observar que el $75.6 \%$ de encuestados pertenecen al género femenino, mientras que $24.4 \%$ corresponde al masculino.

TABLA 2: PASTILLAS O PILDORAS

\begin{tabular}{|cc|c|c|c|c|}
\hline & Frecuencia & Porcentaje & $\begin{array}{c}\text { Porcentaje } \\
\text { válido }\end{array}$ & $\begin{array}{c}\text { Porcentaje } \\
\text { acumulado }\end{array}$ \\
\hline Válidos & ni mala, ni buena & 57 & 22.4 & 22.4 & 22.4 \\
muy mala & 23 & 9.1 & 9.1 & 31.5 \\
algo mala & 43 & 16.9 & 16.9 & 48.4 \\
algo buena & 95 & 37.4 & 37.4 & 85.8 \\
muy buena & 36 & 14.2 & 14.2 & 100.0 \\
Total & 254 & 100.0 & 100.0 & \\
\hline
\end{tabular}

La tabla 2 nos muestra que las pastillas o píldoras son consideradas "algo buena" por parte del $37.4 \%$ de los entrevistados, seguido por el 22,4\% quienes consideran que no es "ni mala ni buena"; el 16,9 \% considera que es "algo mala", el 14,2 \% opina que es "muy buena" en contraste con el 9,1\% que considera que las pastillas o píldoras anticonceptivas son "muy malas".

TABLA 3: Condones o preservativos

\begin{tabular}{|c|c|c|c|c|c|}
\hline & & Frecuencia & Porcentaje & $\begin{array}{l}\text { Porcentaje } \\
\text { válido }\end{array}$ & $\begin{array}{l}\text { Porcentaje } \\
\text { acumulado }\end{array}$ \\
\hline \multirow[t]{6}{*}{ Válidos } & ni mala, ni buena & 32 & 12.6 & 12.6 & 12.6 \\
\hline & muy mala & 21 & 8.3 & 8.3 & 20.9 \\
\hline & algo mala & 20 & 7.9 & 7.9 & 28.7 \\
\hline & algo buena & 88 & 34.6 & 34.6 & 63.4 \\
\hline & muy buena & 93 & 36.6 & 36.6 & $\mid 100.0$ \\
\hline & Total & 254 & 100.0 & 100.0 & \\
\hline
\end{tabular}

La tabla 3, muestra que la opinión sobre los condones o preservativos es "muy buena" por parte del $36.6 \%$ de los entrevistados, seguido muy de cerca por el $34.6 \%$ quienes consideran que es "algo buena"; el 12,6 \% considera que es no es "ni mala, ni buena", del mismo modo, el $8.3 \%$ opina que es "muy mala" apoyado por el $7.9 \%$ de los entrevistados quienes tienen una opinión acerca de los condones o preservativos como "algo mala".

TABLA 4: Óvulos, jaleas, espumas o diafragma

\begin{tabular}{|c|c|c|c|c|c|}
\hline & & Frecuencia & Porcentaje & \begin{tabular}{|l|} 
Porcentaje \\
válido
\end{tabular} & $\begin{array}{l}\text { Porcentaje } \\
\text { acumulado }\end{array}$ \\
\hline \multirow[t]{2}{*}{ Válidos } & ni mala, ni buena & 113 & 44.5 & 44.5 & 44.5 \\
\hline & muy mala & 29 & 11.4 & 11.4 & 55.9 \\
\hline
\end{tabular}




\begin{tabular}{|l|l|l|l|l|} 
algo mala & 51 & $\mid 20.1$ & 20.1 & 76.0 \\
algo buena & 45 & 17.7 & 17.7 & 93.7 \\
$\begin{array}{l}\text { muy buena } \\
\text { Total }\end{array}$ & 16 & 6.3 & 6.3 & 100.0 \\
254 & 100.0 & 100.0 & \\
\hline
\end{tabular}

En cuanto a los resultados obtenidos en la tabla 4, notamos que los óvulos, jaleas, espumas o diafragmas son consideradas "ni malas, ni buenas" por parte del $44.5 \%$ de los entrevistados, seguido por el $20,1 \%$ quienes consideran que es "algo mala"; el 17,7 $\%$ considera que es "algo buena", el 11,4\% opina que es "muy mala" en contraste con el $6.3 \%$ que considera que los óvulos, jaleas, espumas o diafragmas son "muy buena".

TABLA 5: Dispositivos intrauterinos

\begin{tabular}{|c|c|c|c|c|c|}
\hline & & Frecuencia & Porcentaje & $\begin{array}{l}\text { Porcentaje } \\
\text { válido }\end{array}$ & $\begin{array}{l}\text { Porcentaje } \\
\text { acumulado }\end{array}$ \\
\hline \multirow[t]{6}{*}{ Válidos } & ni mala, ni buena & 67 & 26.4 & 26.4 & 26.4 \\
\hline & muy mala & 21 & 8.3 & 8.3 & 34.6 \\
\hline & algo mala & 43 & $\mid 16.9$ & 16.9 & 51.6 \\
\hline & algo buena & 80 & 31.5 & 31.5 & 83.1 \\
\hline & muy buena & 43 & $\mid 16.9$ & 16.9 & $\mid 100.0$ \\
\hline & Total & 254 & $\mid 100.0$ & 100.0 & \\
\hline
\end{tabular}

En cuanto a la tabla anterior, la opinión por parte de los entrevistados es que un $31.5 \%$ considera que los dispositivos intrauterinos son "algo buena", seguido por el $26.4 \%$ quienes consideran que no "ni mala ni buena"; el 16,9 \% considera que es "algo mala"; en contraste con el mismo porcentaje $(16.9 \%)$ considera que es "muy buena" y por último $8.3 \%$ que considera que los dispositivos intrauterinos son "muy malas".

TABLA 6: Hormonas inyectables

\begin{tabular}{|ll|l|l|l|l|}
\hline & & Frecuencia & Porcentaje & $\begin{array}{l}\text { Porcentaje } \\
\text { válido }\end{array}$ & $\begin{array}{l}\text { Porcentaje } \\
\text { acumulado }\end{array}$ \\
\hline Válidos & ni mala, ni buena & 84 & 33.1 & 33.1 & 33.1 \\
& muy mala & 24 & 9.4 & 9.4 & 42.5 \\
& algo mala & 36 & 14.2 & 14.2 & 56.7 \\
algo buena & $\mid 76$ & 29.9 & 29.9 & 86.6 \\
muy buena & 34 & 13.4 & 13.4 & 100.0 \\
Total & 254 & 100.0 & 100.0 & \\
\hline
\end{tabular}

Según la tabla 6, las hormonas inyectables son consideradas "ni mala, ni buena" por parte del $33.1 \%$ de los entrevistados, seguido por el $29,9 \%$ quienes consideran que es "algo buena"; el 14,2 \% considera que es "algo mala", seguido muy de cerca por el 13.4 $\%$ quienes opinan que su opinión acerca de las hormonas inyectables es "muy buena" y por último el 9,4\% que considera que las hormonas inyectables son "muy malas". 
TABLA 7: Operación de la mujer o salpingoclasia o ligadura

\begin{tabular}{|ll|l|l|l|l|}
\hline & Frecuencia & Porcentaje & $\begin{array}{l}\text { Porcentaje } \\
\text { válido }\end{array}$ & $\begin{array}{l}\text { Porcentaje } \\
\text { acumulado }\end{array}$ \\
\hline Válidos & ni mala, ni buena & 44 & 17.3 & 17.3 & 17.3 \\
& muy mala & 14 & 5.5 & 5.5 & 22.8 \\
algo mala & $\mid 20$ & 7.9 & 7.9 & 30.7 \\
algo buena & $\mid 69$ & 27.2 & $\mid 27.2$ & 57.9 \\
$\begin{array}{c}\text { muy buena } \\
\text { Total }\end{array}$ & 107 & 42.1 & $\mid 42.1$ & 100.0 \\
\hline
\end{tabular}

En lo que respecta a la opinión por parte de los entrevistados sobre operación de la mujer, salpingoclasia o ligadura (tabla 7), se encontró que un $42.1 \%$ considera que la ligadura de trompas es "muy buena", seguido por el $27.2 \%$ quienes consideran que es "algo buena"; el 17,3 \% considera que no es "ni buena, ni mala" y por último, $7.9 \%$ considera que es "algo mala" seguido muy de cerca por el $5.5 \%$ que considera la ligadura de trompas es "muy mala".

TABLA 8: Operación del hombre o vasectomía

\begin{tabular}{|ll|l|l|l|l|}
\hline & Frecuencia & Porcentaje & $\begin{array}{l}\text { Porcentaje } \\
\text { válido }\end{array}$ & $\begin{array}{l}\text { Porcentaje } \\
\text { acumulado }\end{array}$ \\
\hline Válidos & ni mala ni buena & 38 & 15.0 & 15.0 & 15.0 \\
& muy mala algo & 21 & 8.3 & 8.3 & 23.2 \\
mala algo buena & 16 & 6.3 & 6.3 & $\mid 29.5$ \\
muy buena & 53 & 20.9 & 20.9 & 50.4 \\
Total & 126 & 49.6 & 49.6 & 100.0 \\
& 254 & 100.0 & 100.0 & \\
\hline
\end{tabular}

En cuanto a la tabla 8, la opinión por parte de los entrevistados es que un $49.6 \%$ considera que la vasectomía es "muy buena", seguido por el $20.9 \%$ quienes consideran que es "algo buena"; el $15.0 \%$ considera que no es "ni buena, ni mala" y por último, el $8.3 \%$ considera que es "muy mala" seguido muy de cerca por el $6.3 \%$ que considera la vasectomía es "algo mala".

TABLA 9: Ritmo, calendario, abstinencia

\begin{tabular}{|ll|l|l|l|l|}
\hline & Frecuencia & Porcentaje & $\begin{array}{l}\text { Porcentaje } \\
\text { válido }\end{array}$ & $\begin{array}{l}\text { Porcentaje } \\
\text { acumulado }\end{array}$ \\
\hline Válidos & ni mala ni buena & 45 & 17.7 & 17.7 & 17.7 \\
& muy mala algo & 89 & 35.0 & 35.0 & 52.8 \\
mala algo buena & 53 & 20.9 & 20.9 & 73.6 \\
muy buena & 31 & 12.2 & 12.2 & 85.8 \\
Total & 36 & 14.2 & 14.2 & 100.0 \\
& 254 & 100.0 & 100.0 & \\
\hline
\end{tabular}


La tabla 9, muestra que se encontró que un $35.0 \%$ considera que el método de ritmo, calendario o abstinencia es "muy mala", seguido por el $20.9 \%$ quienes consideran que es "algo mala"; el 17,7 \% considera que no es "ni buena, ni mala" y por último, $14.2 \%$ considera que es "muy buena" seguido muy de cerca por el $12.2 \%$ que considera al método de ritmo, calendario y la abstinencia como "algo buena".

TABLA 10: Retirada o interrupción

\begin{tabular}{|ll|l|l|l|l|}
\hline & Frecuencia & Porcentaje & $\begin{array}{l}\text { Porcentaje } \\
\text { válido }\end{array}$ & $\begin{array}{l}\text { Porcentaje } \\
\text { acumulado }\end{array}$ \\
\hline Válidos & ni mala ni buena & 50 & 19.7 & 19.7 & 19.7 \\
& $\begin{array}{l}\text { muy mala algo } \\
\text { mala algo buena }\end{array}$ & 133 & 52.4 & 52.4 & 72.0 \\
muy buena & 37 & 14.6 & 14.6 & 86.6 \\
Total & 21 & 8.3 & 8.3 & 94.9 \\
& 13 & 5.1 & 5.1 & 100.0 \\
& 254 & 100.0 & 100.0 & \\
\hline
\end{tabular}

Igualmente, la tabla 10 muestra que los entrevistados en un $52.4 \%$ consideran que el método de retirada o interrupción es "muy mala", seguido por el $19.7 \%$ quienes piensan que no es "ni mala, ni buena"; el 14,6\% considera que es "algo mala" y por último, $8.3 \%$ considera que es "algo buena" seguido muy de cerca por el $5.1 \%$ que considera la retirada o interrupción como "muy buena".

TABLA 11: Implantes

\begin{tabular}{|ll|l|l|l|l|}
\hline & Frecuencia & Porcentaje & $\begin{array}{l}\text { Porcentaje } \\
\text { válido }\end{array}$ & $\begin{array}{l}\text { Porcentaje } \\
\text { acumulado }\end{array}$ \\
\hline Válidos & ni mala ni buena & 79 & 31.1 & 31.1 & 31.1 \\
& muy mala & 42 & 16.5 & 16.5 & 47.6 \\
algo mala & 52 & 20.5 & 20.5 & 68.1 \\
algo buena & 59 & 23.2 & 23.2 & 91.3 \\
muy buena & 22 & 8.7 & 8.7 & 100.0 \\
Total & 254 & 100.0 & 100.0 & \\
\hline
\end{tabular}

En la opinión por parte de los entrevistados sobre los implantes (tabla N 11), se encontró que un $31.1 \%$ considera que los implantes subdérmicos son "ni mala, ni buena", seguido por el $23.2 \%$ quienes consideran que es "algo buena"; el $20.5 \%$ considera que es "algo mala" y por último, $16.5 \%$ considera que es "muy mala" seguido muy de cerca por el 8.7 $\%$ que considera que su opinión acerca de los implantes subdérmicos es "muy buena".

TABLA 12: Pastillas de emergencia

\begin{tabular}{|ll|l|l|l|l|}
\hline & & & $\begin{array}{l}\text { Porcentaje } \\
\text { válido }\end{array}$ & $\begin{array}{l}\text { Porcentaje } \\
\text { acumulado }\end{array}$ \\
\hline Válidos & Frecuencia & Porcentaje & \\
& mula ni buena & 62 & 24.4 & 24.4 & 24.4 \\
& 54 & 21.3 & 21.3 & 45.7 \\
& 51 & 20.1 & 20.1 & 65.7
\end{tabular}




\begin{tabular}{|l|l|l|l|l|} 
mala algo buena & 52 & 20.5 & 20.5 & 86.2 \\
muy buena & 35 & 13.8 & 13.8 & 100.0 \\
Total & 254 & 100.0 & 100.0 & \\
\hline
\end{tabular}

De acuerdo a la tabla anterior (tabla 12), la opinión por parte de los entrevistados es que un $24.4 \%$ considera que las pastillas de emergencia es "ni mala, ni buena", seguido por el $21.3 \%$ quienes consideran que es "muy mala"; el $20.5 \%$ considera que es "algo buena" y por último, $20.1 \%$ considera que es "algo mala" seguido por el $13.8 \%$ que considera que las pastillas de emergencia es "muy buena".

TABLA 13: Opinión general de todos los métodos

\begin{tabular}{|ll|l|l|l|l|}
\hline & Frecuencia & Porcentaje & $\begin{array}{l}\text { Porcentaje } \\
\text { válido }\end{array}$ & $\begin{array}{l}\text { Porcentaje } \\
\text { acumulado }\end{array}$ \\
\hline Válidos & ni mala, ni buena & 97 & 38.2 & 38.2 & 38.2 \\
& $\begin{array}{l}\text { muy mala algo } \\
\text { mala algo buena }\end{array}$ & 13 & 5.1 & 5.1 & 43.3 \\
muy buena & 12 & 4.7 & 4.7 & 48.0 \\
Total & 105 & 41.3 & 41.3 & 89.4 \\
& 27 & 10.6 & 10.6 & 100.0 \\
& 254 & 100.0 & 100.0 & \\
\hline
\end{tabular}

Según la tabla anterior, la opinión por parte de los entrevistados es que un $41.3 \%$ tiene una opinión general de todos los métodos como "algo buena", seguido por el $38.2 \%$ quienes consideran que es "ni mala, ni buena"; el $10.6 \%$ considera que es "muy buena" y por último, $5.1 \%$ considera que es "muy mala" seguido muy de cerca por el $4.7 \%$ que considera su opinión general de todos los métodos anticonceptivos es "algo mala".

\section{DISCUSIÓN}

El objetivo de la presente investigación fue el de determinar cuáles eran las opiniones de los estudiantes del programa de Medicina de la Universidad del Magdalena mediante el autodiligenciamiento de encuestas sobre métodos anticonceptivos, lo cual mostró que la vasectomía y la ligadura de trompas eran las que más demandas presentaban, seguido por el preservativos y las pastillas. Esto es similar a lo reportado por Santoyo y colaboradores, quienes encontraron que de los métodos anticonceptivos más usados se encuentra el condón y las pastillas o píldoras por parte de las mujeres (11); así mismo Pérez y colaboradores muestran que el método anticonceptivo más utilizado es el condón, referido por un $76.8 \%$ de las entrevistadas (14).

Los resultados de Carvajal expresan que la anticoncepción de urgencia la han utilizado un $35,6 \%$ de las usuarias alguna vez como método anticonceptivo (15). De igual forma, Ospina determina que se maneja un conocimiento pobre e inadecuado con respecto a la indicación, cómo se emplea, frecuencia de uso y las contraindicaciones (6). En contraste, con el actual estudio, no habría punto de comparación por no conocer sobre el uso de éste, pero las opiniones que se tiene al respecto se diversifican. 
En el estudio realizado por Morales y colaboradores revelan un nivel de conocimientos bajo acerca de los métodos anticonceptivos $(39,7 \%)$, aunque la opinión general de los métodos anticonceptivos es favorable (16).

Con respecto a los anticonceptivos más favorables para su uso como el condón y las pastillas en el actual estudio, en contraste con el estudio de Vásquez y colaboradores si destacan que el preservativo es de mayor uso seguido por los anticonceptivos inyectables que varía con los anticonceptivos orales como se muestra en el presente (17).

En opinión general, nuestros entrevistados consideran de forma positiva los métodos anticonceptivos de barrera, definitivos y hormonales, no obstante, en el estudio de Artehotua y colaboradores se compara que el nivel más alto de conocimiento está en los métodos de barrera y naturales y el nivel más bajo en los métodos hormonales y quirúrgicos, por lo cual no se podría comparar el grado de estos en el presente estudio (18).

\section{CONCLUSIONES}

En términos concluyentes la población universitaria del programa de Medicina observan de manera favorable los métodos anticonceptivos por lo cual es relativamente coherente con el conocimiento y uso de estos en su práctica sexual ya que han resultado efectivos para reducir fenómenos como embarazos no deseados e infecciones de transmisión sexual.

A pesar de tenerse la certeza del conocimiento sobre el objetivo de estudio, se desconoce si estos son adquiridos correctamente o existe la posibilidad de falencia en algunos y por tanto, optan por los más habituales entre la población universitaria de edad promedio de 20 como el condón y las pastillas anticonceptivas. Se determina, a su vez, que aquellos métodos anticonceptivos naturales son conocidos y la opinión que surge de ellos, sugiere la posibilidad que la práctica de estos es nula o poco recomendable. Por otro lado, los métodos anticonceptivos definitivos los consideran positivos, por lo cual sugeriría que los reconocen como aquellos que podrían radicalmente reducir las tasas de embarazos no deseados.

Por otra parte, sin lugar a duda, se podría complementar en este estudio variables como el uso de los métodos anticonceptivos, incluir muestras de población de otros programas de la universidad, consulta sobre la opinión de cuál sería más seguro y/o efectivo para reducir problemáticas de salud y sociodemográficas como los casos de infecciones de transmisión sexual y las tasas de embarazos en población adulta joven y sobre cuáles fueron los medios por los cuales tienen dicha opinión.

\section{BIBLIOGRAFÍA}


1. El Heraldo. Embarazos no deseados. (2011). disponible en: http://www.elheraldo.co/editorial/embarazos-no-deseados-30367

2. Profamilia. Encuesta nacional de demografía y salud-ENDS Colombia 2010. disponible en: http://www.profamilia.org.co/encuestas/Profamilia/Profamilia/images/stories/documentos/Pri ncipales indicadores.pdf

3. PROFAMILIA. Encuesta Nacional de Demografía y Salud, principales indicadores. Disponible en:

http://www.profamilia.org.co/encuestas/Profamilia/Profamilia/images/stories/documentos/Pri ncipales indicadores.pdf. Consultado el 20 de febrero de 2013.

4. Fernandez R. Lopez L. Martinez H. Kopecky D. Uzcategui G. Muñoz M. Métodos anticonceptivos: orevalencia de uso según método en el hospital "materno del este", petare. Rev Obstet Ginecol. 2009; 69(1): 28-34.

5. Tapia A. Villaseñor B. Nuño B. Conocimientos y actitudes hacia el uso de la anticoncepción de emergencia en jóvenes universitarios. Rev Med Inst Mex Seguro. 2008; 46 (1): 33-41.

6. Ospina J. Manrique F. Prácticas y comportamientos sexuales en estudiantes universitarios. Av. Enferm. 2007; XXV (2): 101-111.

7. Gómez P. Arguello K. Cáceres C. Gutiérrez E. Rodríguez A. et al. Anticoncepción de emergencia hormonal: conocimientos, actitudes y prácticas en estudiantes universitarias.

Estudio de corte trasversal. Colombia. 2008; 5. disponible en: http://www.uelbosque.edu.co/sites/default/files/publicaciones/revistas/revista colombiana e nfermeria/volumen5/anticoncepcion emergencia hormonal.pdf

8. Cáceres G. Echavarria M. Ghilarducci C. Pomares D. Nivel de conocimientos sobre métodos anticonceptivos como futuros profesionales de la salud en estudiantes del último año de medicina. Rev. Posgrado de la Via cátedra de medicina. 2009; 192. disponible en: http://med.unne.edu.ar/revista/revista192/2 192.pdf

9. Abeldaño R. Sacchi M. Comportamientos sexuales y uso de anticonceptivos en estudiantes universitarios. Rev. Facultad ciencias de la salud. 2011; 1 (1).

10. Navarro V. Alvarez J. Actitudes y comportamientos en torno a la anticoncepción y el embarazo en una muestra de estudiantes universitarios. Rev. Psicología y Humanidades. 2012; disponible en: http://www.eepsys.com/es/actitudes-comportamientos-entornolaanticoncepcion-el-embarazo-en-una-muestra-de-estudiantes-universitarios/

11. Santoyo S. Ponce E. Gómez F. Fernández M. Domínguez J. Díaz V. Confiabilidad y validez de constructo de una escala para evaluar las actitudes y opiniones hacia las prácticas anticonceptivas en 14 países de América Latina. Revista de la Facultad de Medicina de la UNAM 2010; 53 (4): 15-22.

12. Ministerio de la protección social. Ley 1090 del 2006. Ministerio de la protección social. 2006; Disponible en: http://www.upb.edu.co/pls/portal/docs/PAGE/GPV2 UPB MEDELLIN/PGV2 M030 PRERA DOS/PGV2 M030040020 PSICOLOGIA/CODIGO ETICOCODIGO\%20DEONTOLOGICO $\% 20 Y \% 20 B I O E T I C O . P D F$

13. Ministerio de salud. Título II: De la investigación en seres humanos. 2013; Recuperado el 2002-2014, disponible en: http://www.dib.unal.edu.co/promocion/etica res 8430 1993.pdf

14. Pérez I. Lie A. Pérez J. \& Hernández I. Empleo de métodos anticonceptivos en adolescentes escuela internacional de educación física y deporte. Rev. Ciencias Médicas de la Habana. 2009; 2 (15).

15. Carvajal J. Maroto A. Conocimiento y opiniones sobre la Píldora Anticonceptiva de Emergencia. Asociación demográfica costarricense. Programa de reproducción planeada. 2003.

16. Morales E. Solanelles A. Gonzales S. \& Miranda O. Embarazo no deseado en alumnas universitarias. Revista Cubana de Medicina Militar. 2013; 42 (2). 
17. Vásquez M. Romero A. Rivas A. Conocimientos, Actitudes y Prácticas en salud sexual entre los estudiantes de los primeros semestres de enfermería. Revista de la Facultad de Ciencias de la Salud DUAZARY. 2010; 7 (2): 211-218.

18. Artehotua W. Acosta S. López A. Suárez J. Melo A. Carrascal S. Conocimiento métodos anticonceptivos en estudiantes de pregrado. Revista Ciencia y Cuidado. 2011; 8 (1). 